Internat. J. Math. \& Math. Sci.

Vol. 22, No. 3 (1999) 543-546

S 0161-1712〈99 $22543-4$

(C) Electronic Publishing House

\title{
INEQUALITIES VIA CONVEX FUNCTIONS
}

\section{A. ABOU-TAIR and W. T. SULAIMAN}

(Received 25 May 1998)

\begin{abstract}
A general inequality is proved using the definition of convex functions. Many
\end{abstract} major inequalities are deduced as applications.

Keywords and phrases. Convex functions, inequalities.

1991 Mathematics Subject Classification. 26A51, 26D15.

1. Introduction. Kapur and Kumer (1986) have used the principle of dynamical programming to prove major inequalities due to Shannon, Renyi, and Hölder. See [1]. In this note, we prove a general inequality using convex functions. As a result, the inequalities of Shannon, Renyi, Hölder, and others are all deduced.

Let $I$ be an interval in $\mathbb{R}, f: I \rightarrow \mathbb{R}$ is said to be convex if and only if, for all $x, y \in I$, all $\lambda, 0 \leq \lambda \leq 1$,

$$
f[\lambda x+(1-\lambda) y] \leq \lambda f(x)+(1-\lambda) y .
$$

Here, we give the following new definitions:

(a) Let $f$ and $g$ be two functions and let $I$ be an interval in $\mathbb{R}$ for which $f \circ g$ is defined, then $f$ is said to be $g$-convex if and only if, for all $x, y \in I$, all $\lambda$, $0 \leq \lambda \leq 1$,

$$
f[\lambda g(x)+(1-\lambda) g(y)] \leq \lambda f \circ g(x)+(1-\lambda) f \circ g(y) .
$$

(b) If the inequality is reversed, then $f$ is said to be $g$-concave.

If $g(x)=x$, the two definitions of $g$-convex and convex functions become identical.

THEOREM 1.1. Let $f$ be $g$-convex, then

(i) if $g$ is linear, then $f \circ g$ is convex, and

(ii) if $f$ is increasing and $g$ is convex, then $f \circ g$ is convex.

\section{Proof.}

(i)

$$
\begin{aligned}
f \circ g[\lambda x+(1-\lambda) y] & =f[\lambda g(x)+(1-\lambda) g(y)] \\
& \leq \lambda f \circ g(x)+(1-\lambda) f \circ g(y) .
\end{aligned}
$$

(ii)

$$
\begin{aligned}
f \circ g[\lambda x+(1-\lambda) y] & \leq f[\lambda g(x)+(1-\lambda) g(y)] \\
& \leq \lambda f \circ g(x)+(1-\lambda) f \circ g(y) .
\end{aligned}
$$


LEMMA 1.1. Let $f$ be $g$-convex and let $\sum_{i=1}^{n} t_{i}=T_{n}=1, t_{i} \geq 0, i=1,2, \ldots, n$, then

$$
f\left(\sum_{i=1}^{n} t_{i} g\left(x_{i}\right)\right) \leq \sum_{i=1}^{n} t_{i} f \circ g\left(x_{i}\right) .
$$

ProOF.

$$
\begin{aligned}
f\left(\sum_{i=1}^{n} t_{i} g\left(x_{i}\right)\right) & =f\left(T_{n-1} \sum_{i=1}^{n-1} \frac{t_{i}}{T_{n-1}} g\left(x_{i}\right)+t_{n} g\left(x_{n}\right)\right) \\
& \leq T_{n-1} f\left(\sum_{i=1}^{n-1} \frac{t_{i}}{T_{n-1}} g\left(x_{i}\right)\right)+t_{n} f \circ g\left(x_{n}\right) \\
& =T_{n-2} f\left(\frac{T_{n-2}}{T_{n-1}} \sum_{i=1}^{n-2} \frac{t_{i}}{T_{n-2}} g\left(x_{i}\right)+\frac{t_{n-1}}{T_{n-1}} g\left(x_{n-1}\right)\right)+t_{n} f \circ g\left(x_{n}\right) \\
& \leq T_{n-2} f\left(\sum_{i=1}^{n-2} \frac{t_{i}}{T_{n-2}} g\left(x_{i}\right)\right)+t_{n-1} f \circ g\left(x_{n-1}\right)+t_{n} f \circ g\left(x_{n}\right) \\
& \vdots \\
& \leq \sum_{i=1}^{n} t_{i} f \circ g\left(x_{i}\right) .
\end{aligned}
$$

LEMMA 1.2. For any function $g$, the exponential function $f(x)=e^{x}$ is $g$-convex.

Proof. Define

$$
F(x)=\lambda e^{g(x)}+(1-\lambda) e^{g(y)}-e^{\lambda g(x)+(1-\lambda) g(y)} .
$$

Let

$$
G(t)=(1-\lambda)+\lambda t-t^{\lambda}, \quad t>0
$$

It follows that

$$
G^{\prime}(t)=\lambda\left(1-t^{\lambda-1}\right), \quad G^{\prime \prime}(t)=\lambda(1-\lambda) t^{\lambda-2} .
$$

Thus, $G^{\prime}(t)=0$ when $t=1$ and $G^{\prime \prime}(1)=\lambda(1-\lambda)>0$. Hence, $G$ has its minimum value 0 at $t=1$ and this implies $G(t) \geq 0, t>0$. The result follows by putting $F(x)=$ $e^{g(y)} G\left(e^{g(x)-g(y)}\right)$.

COROLlary 1.3. The function $f(x)=\ln (x)$ is concave for if $h(x)=e^{x}$, then, by Lemma 1.2, $h$ is $f$-convex. Hence,

$$
e^{\lambda(\ln x)+(1-\lambda) \ln y} \leq \lambda e^{\ln x}+(1-\lambda) e^{\ln y}=\lambda x+(1-\lambda y) .
$$

It follows that

$$
\lambda \ln x+(1-\lambda) \ln y \leq \ln [\lambda x+(1-\lambda) y]
$$

\section{Main inequality}

THEOREM 2.1.

$$
\sum_{j=1}^{n} \prod_{i=1}^{m}\left(p_{i j}\right)^{q_{i} / \sum_{i=1}^{m} q_{i}} \leq \frac{\sum_{i=1}^{m} \sum_{j=1}^{n} p_{i j} q_{i}}{\sum_{i=1}^{m} q_{i}} .
$$

Proof. If $f(x)=e^{x}$ and $g(x)=\ln x$, then $f$ is $g$-convex. By Lemma 1.2, we have 


$$
\begin{aligned}
\prod_{i=1}^{m}\left(p_{i j}\right)^{q_{i} / \sum_{i=1}^{m} q_{i}} & =e^{\ln \left(\prod_{i=1}^{m}\left(p_{i j}\right)^{q_{i}} / \sum_{i=1}^{m} q_{i}\right)} \\
& =e^{\sum_{i=1}^{m} \ln \left(p_{i j}\right)^{q_{i}} / \sum_{i=1}^{m} q_{i}}=e^{\sum_{i=1}^{m}\left(q_{i} / \sum_{i=1}^{m} q_{i}\right) \ln p_{i j}} \\
& \leq \sum_{i=1}^{m}\left(\frac{q_{i}}{\sum_{i=1}^{m} q_{i}}\right) e^{\ln p_{i j}}=\frac{\sum_{i=1}^{m} q_{i} p_{i j}}{\sum_{i=1}^{m} q_{i}} .
\end{aligned}
$$

Therefore,

$$
\sum_{j=1}^{n} \prod_{i=1}^{m}\left(p_{i j}\right)^{q_{i} / \sum_{i=1}^{m} q_{i}} \leq \frac{\sum_{j=1}^{n} \sum_{i=1}^{m} p_{i j} q_{i}}{\sum_{i=1}^{m} q_{i}}=\frac{\sum_{i=1}^{m} \sum_{j=1}^{n} p_{i j} q_{i}}{\sum_{i=1}^{m} q_{i}}
$$

\section{Applications}

THEOREM 3.1 (Shannon's inequality). Given $\sum_{i=1}^{m} a_{i}=a, \sum_{i=1}^{m} b_{i}=b$, then

$$
a \ln \left(\frac{a}{b}\right) \leq \sum_{i=1}^{m} a_{i} \ln \left(\frac{a_{i}}{b_{i}}\right), \quad a_{i}, b_{i} \geq 0 .
$$

Proof. Applying Theorem 2.1 by putting

$$
p_{i j}=\frac{b_{i}}{a_{i}}, \quad j=1, \quad q_{i}=a_{i}, \quad \sum_{i=1}^{m} a_{i}=a, \quad \sum_{i=1}^{m} b_{i}=b,
$$

we have

$$
\prod_{i=1}^{m}\left(\frac{b_{i}}{a_{i}}\right)^{a_{i} / \sum_{i=1}^{m} a_{i}} \leq \frac{\sum_{i=1}^{m} b_{i}}{\sum_{i=1}^{m} a_{i}}
$$

That is

$$
\prod_{i=1}^{m}\left(\frac{b_{i}}{a_{i}}\right)^{a_{i} / a} \leq \frac{b}{a}
$$

It follows that

$$
\frac{a}{b} \leq \prod_{i=1}^{m}\left(\frac{a_{i}}{b_{i}}\right)^{a_{i} / a}
$$

Hence, we get

$$
a \ln \left(\frac{a}{b}\right) \leq \sum_{i=1}^{m} a_{i} \ln \left(\frac{a_{i}}{b_{i}}\right)
$$

TheOrem 3.2 (Renyi's inequality). Given $\sum_{i=1}^{m} a_{i}=a, \sum_{i=1}^{m} b_{i}=b$, then, for $\alpha>0$, $\alpha \neq 1$,

$$
\frac{1}{\alpha-1}\left(a^{\alpha} b^{1-\alpha}-a\right) \leq \sum_{i=1}^{m} \frac{1}{\alpha-1}\left(a_{i}^{\alpha} b_{i}^{1-\alpha}-a_{i}\right), \quad a_{i}, b_{i} \geq 0 .
$$

Proof. Applying Theorem 2.1 with $i=2, p_{1 j}=c_{j}, p_{2 j}=d_{j}, q_{1}=\lambda, q_{2}=1-\lambda$, $0<\lambda<1$, we have

$$
\sum_{j=1}^{m} c_{j}^{\lambda} d_{j}^{1-\lambda} \leq \sum_{j=1}^{m}\left(\lambda c_{j}+(1-\lambda) d_{j}\right)
$$


On putting $c_{j}=\left(a_{j} / \sum_{j=1}^{m} a_{j}\right)$ and $d_{j}=\left(b_{j} / \sum_{j=1}^{m} b_{j}\right)$, inequality (22) implies

$$
\sum_{j=1}^{m} a_{j}^{\lambda} b_{j}^{1-\lambda} \leq\left(\sum_{j=1}^{m} a_{j}\right)^{\lambda}\left(\sum_{j=1}^{m} b_{j}\right)^{1-\lambda},
$$

and this gives

$$
\frac{a^{\lambda} b^{1-\lambda}}{\lambda-1} \leq \frac{1}{\lambda-1} \sum_{j=1}^{m} a_{j}^{\lambda} b_{j}^{1-\lambda} .
$$

Thus, for the case $0<\alpha<1$, the theorem follows from inequality (24) by setting $\lambda=\alpha$. Now, inequality (23) implies

$$
\left(\sum_{j=1}^{m} a_{j}^{\lambda} b_{j}^{1-\lambda}\right)^{1 / \lambda}\left(\sum_{j=1}^{m} b_{j}\right)^{1-1 / \lambda} \leq \sum_{j=1}^{m} a_{j} .
$$

Let $a_{j}^{\lambda} b_{j}^{1-\lambda}=e_{j}, \lambda=1 / \alpha$, then inequality (25) gives

$$
\frac{1}{\alpha-1}\left(\sum_{j=1}^{m} e_{j}\right)^{\alpha}\left(\sum_{j=1}^{m} b_{j}\right)^{1-\alpha} \leq \frac{1}{\alpha-1} \sum_{j=1}^{m} e_{j}^{\alpha} b_{j}^{1-\alpha} .
$$

This completes the proof of the theorem.

THEOREM 3.3 (Generalization of Hölder's inequality).

$$
\sum_{j=1}^{n} \prod_{i=1}^{m}\left(p_{i j}\right)^{q_{i}} \leq \prod_{i=1}^{m}\left(\sum_{j=1}^{n} p_{i j}\right)^{q_{i}}, \quad \sum_{i=1}^{m} q_{i}=1 .
$$

Proof. Applying Theorem 2.1 with $p_{i j} / \sum_{j=1}^{n} p_{i j}$ instead of $p_{i j}$, we get

$$
\sum_{j=1}^{n} \prod_{i=1}^{m}\left(\frac{p_{i j}}{\sum_{j=1}^{n} p_{i j}}\right)^{q_{i}} \leq \sum_{i=1}^{m}\left(\sum_{j=1}^{n}\left(\frac{p_{i j}}{\sum_{j=1}^{n} p_{i j}}\right)\right) q_{i}=\sum_{i=1}^{m} q_{i}=1,
$$

which implies

$$
\sum_{j=1}^{n} \prod_{i=1}^{m}\left(p_{i j}\right)^{q_{i}} \leq \prod_{i=1}^{m}\left(\sum_{j=1}^{n} p_{i j}\right)^{q_{i}}
$$

THEOREM 3.4 (Arithmetic-Geometric-Mean inequality).

$$
\left(\prod_{i=1}^{m} x_{i}\right)^{1 / m} \leq \frac{1}{m} \sum_{i=1}^{m} x_{i}
$$

Proof. Applying Theorem 2.1, with $j=1, p_{i j}=x_{i}, q_{i}=1$.

\section{REFERENCES}

[1] J. N. Kapur, V. Kumar, and U. Kumar, A measure of mutual divergence among a number of probabilty distributions, Internat. J. Math. Math. Sci. 10 (1987), no. 3, 597-607. MR 89d:94030. Zbl 641.94006.

ABOU-TAIR: ZARKA PRIVATE UNIVERSITY, ZARKA, JORDAN

SULAIMAN: P.O. BOX 120054, DOHA, QATAR 


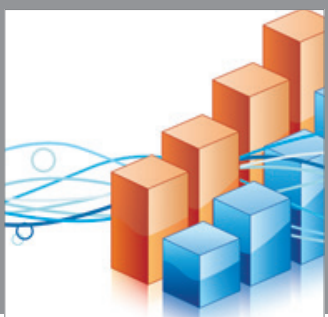

Advances in

Operations Research

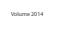

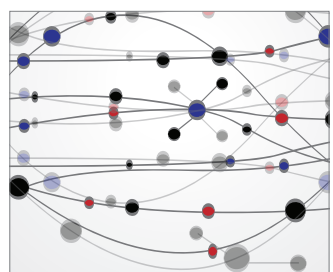

\section{The Scientific} World Journal
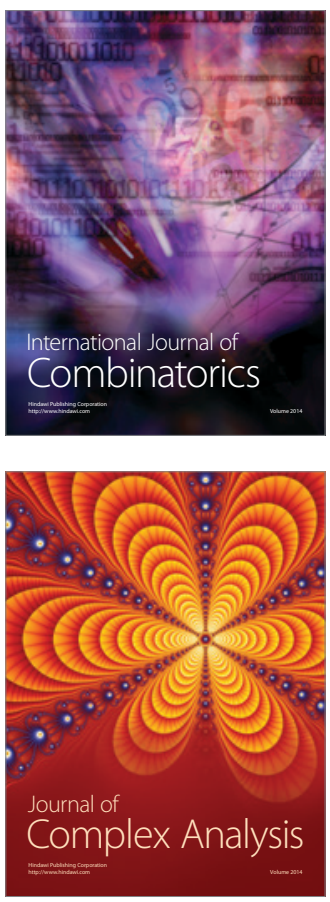

International Journal of

Mathematics and

Mathematical

Sciences
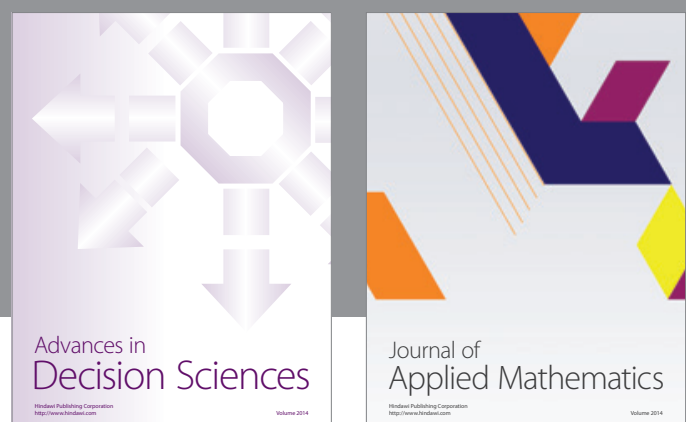

Journal of

Applied Mathematics
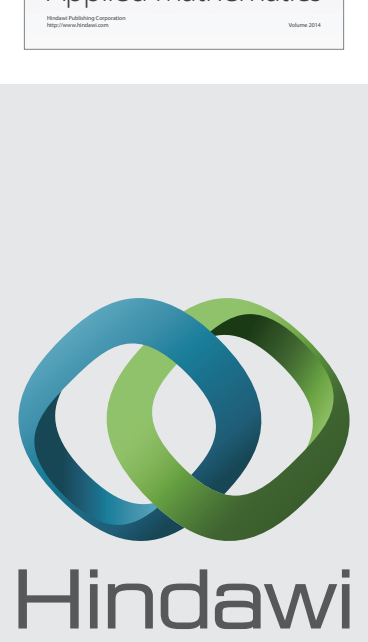

Submit your manuscripts at http://www.hindawi.com
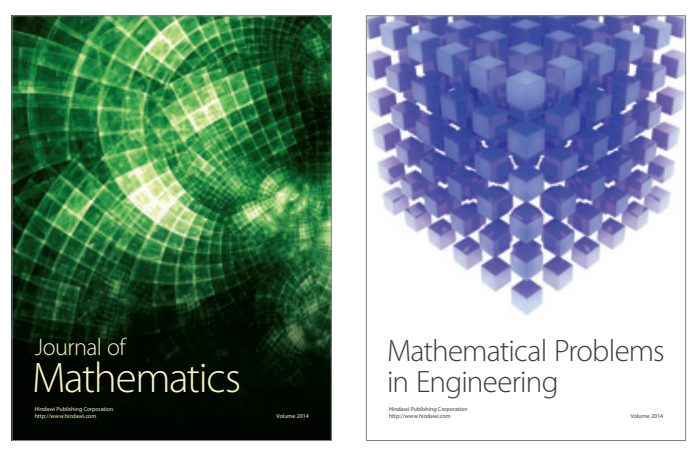

Mathematical Problems in Engineering
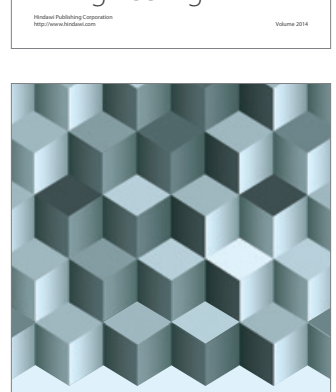

Journal of

Function Spaces
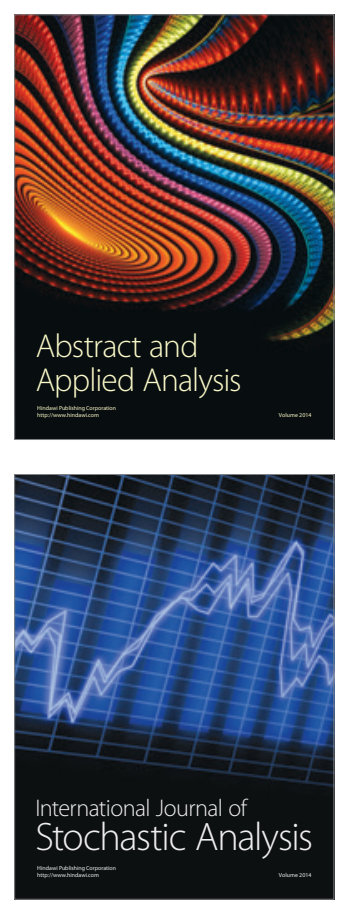

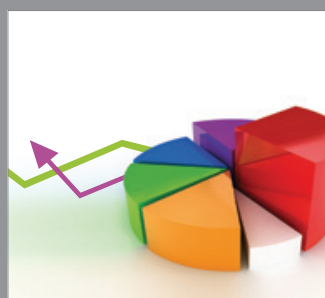

ournal of

Probability and Statistics

Promensencen
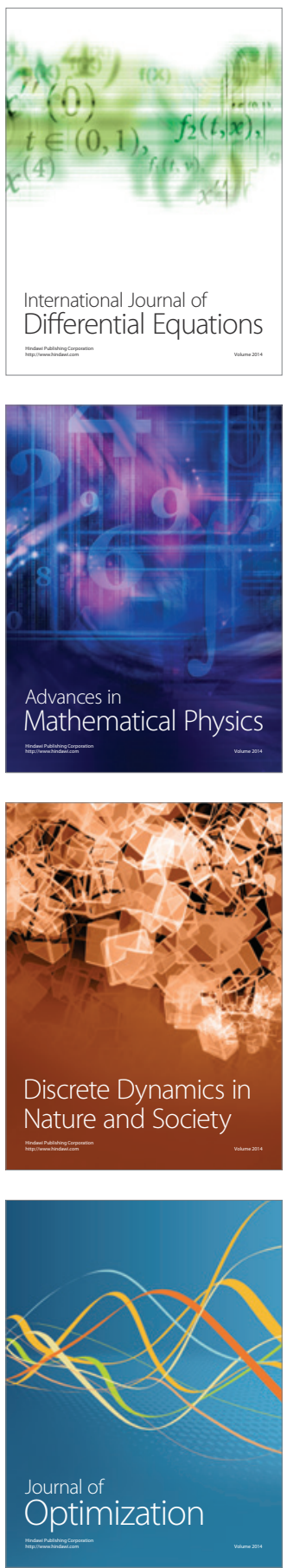